\title{
Treatment with non-selective beta blockers is associated with reduced severity of systemic inflammation and improved survival of patients with acute-on-chronic liver failure ${ }^{\text {is }}$
}

\author{
Rajeshwar P. Mookerjee ${ }^{1}$, Marco Pavesi ${ }^{2}$, Karen Louise Thomsen ${ }^{1}$, Gautam Mehta ${ }^{1}$, \\ Jane Macnaughtan ${ }^{1}$, Flemming Bendtsen ${ }^{3}$, Minneke Coenraad ${ }^{4}$, Jan Sperl ${ }^{5}$, Pere Gines ${ }^{6,7,8,9}$, \\ Richard Moreau ${ }^{10,11,12,13}$, Vicente Arroyo ${ }^{2}$, Rajiv Jalan ${ }^{1, *}$, \\ for the CANONIC Study Investigators of the EASL-CLIF Consortium
}

\begin{abstract}
${ }^{1}$ Liver Failure Group, UCL Institute for Liver and Digestive Health, UCL Medical School, Royal Free Hospital, London, United Kingdom; ${ }^{2}$ European Foundation for the Study of Chronic Liver Failure (EF-CLIF) and EASL-CLIF Consortium; ${ }^{3}$ Department of Gastroenterology, Hvidovre Hospital, University of Copenhagen, Copenhagen, Denmark; ${ }^{4}$ Department of Gastroenterology-Hepatology, Leiden University Medical Center, Leiden, The Netherlands; ${ }^{5}$ Department of Hepatogastroenterology, Institute for Clinical and Experimental Medicine, Prague, Czech Republic;

${ }^{6}$ Liver Unit, Hospital Clínic de Barcelona, University de Barcelona, Barcelona, Spain; ${ }^{7}$ University of Barcelona, Barcelona, Spain; ${ }^{8}$ Institut d'Investigacions Biomediques August Pi I Sunyer (IDIBAPS), Barcelona, Spain; ${ }^{9}$ Centro d'Investigación Biomédica en Red Enfermedades Hepáticas y Digestivas (CIBEReHD), Barcelona, Spain; ${ }^{10}$ Inserm, U1149, Centre de Recherche sur l'Inflammation (CRI), Clichy and Paris, France; ${ }^{11}$ UMRS1149, Université Paris Diderot-Paris 7, Paris, France, ${ }^{12}$ Département Hospitalo-Universitaire (DHU) UNITY, Service d'Hépatologie, Hôpital Beaujon, Assistance Publique-Hôpitaux de Paris, Clichy, France; ${ }^{13}$ Laboratoire d'Excellence Inflamex, PRES Sorbonne Paris Cité, Paris, France
\end{abstract}

See Editorial, pages 532-534

Background \& Aims: Non-selective beta blockers (NSBBs) have been shown to have deleterious outcomes in patients with refractory ascites, alcoholic hepatitis and spontaneous bacterial peritonitis leading many physicians to stop the drug in these cases. Acute-on-chronic liver failure (ACLF) is characterized by systemic inflammation and high mortality. As NSBBs may have beneficial effects on gut motility and permeability and, systemic inflammation, the aims of this prospective, observational study were to determine whether ongoing use of NSBBs reduced 28-day mortality in ACLF patients.

Methods: The study was performed in 349 patients with ACLF included in the CANONIC study, which is a prospective observational investigation in hospitalized cirrhotic patients with acute deterioration. The data about the use of NSBBs, its type and

Keywords: Acute-on-chronic liver failure; Cirrhosis; Multi-organ failure; Sepsis; Prognosis; Non-selective beta blockers.

Received 14 July 2015; received in revised form 14 October 2015; accepted 19 October 2015; available online 28 October 2015

Guest editor: Didier Samuel

DOI of original article: http://dx.doi.org/10.1016/j.jhep.2015.12.012.

* Corresponding author. Address: Liver Failure Group, UCL Institute for Liver and Digestive Health, UCL Medical School, Royal Free Hospital, London, United Kingdom. Tel.: +442074332795.

E-mail address: r.jalan@ucl.ac.uk (R. Jalan).

Abbreviations: ACLF, acute-on-chronic liver failure; AD, acute decompensation; CANONIC study, EASL-CLIF a cute-on-chronic liver failure study; CLIF, chronic liver failure; CLIF-C ACLFs, CLIF consortium ACLF score; CLIF-C OFs, CLIF consortium organ failure score; CLIF-SOFAs, CLIF-sequential organ failure assessment score; CPs, Child-Pugh score; E, epinephrine; EASL, European Association for the Study of the Liver; $\mathrm{FIO}_{2}$, fraction of inspired oxygen; HE, hepatic encephalopathy; INR, international normalized ratio; MAP, mean arterial pressure; MELDs, model of end-stage liver disease; MELD-Nas, MELD-sodium score; NE, norepinephrine, $\mathrm{PaO}_{2}$, partial pressure of arterial oxygen; SOFA, sequential organ failure assessment; $\mathrm{SpO}_{2}$, pulse oximetric saturation. dosage was specifically recorded. Patient characteristics at enrollment significantly associated with treatment and mortality were taken into account as potential confounders to adjust for treatment effect. A logistic regression model was fitted.

Results: 164 (47\%) ACLF patients received NSBBs whereas 185 patients did not. Although the CLIF-C ACLF scores were similar at presentation, more patients in the NSBB treated group had lower grades of ACLF ( $p=0.047)$ at presentation and significantly more patients improved. Forty patients $(24.4 \%)$ died in NSBB treated group compared with 63 patients $(34.1 \%)(p=0.048)$ [estimated risk-reduction 0.596 (95\%CI: $0.361-0.985 ; p=0.0436)$ ]. This improvement in survival was associated with a significantly lower white cell count (NSBB: 8.5 (5.8); no NSBB: 10.8 (6.6); $p=0.002$ ). No long-term improvement in survival was observed. Conclusions: This study shows for the first time that ongoing treatment with NSBBs in cirrhosis is safe and reduces the mortality if they develop ACLF. Careful thought should be given before stopping NSBBs in cirrhotic patients.

(c) 2015 European Association for the Study of the Liver. Published by Elsevier B.V. All rights reserved.

\section{Introduction}

Acute-on-chronic liver failure (ACLF) is a recently defined entity characterized by acute deterioration of liver function, multiorgan failure and high mortality [1-4]. A characteristic feature of this syndrome is systemic inflammation, the severity of which is an independent predictor of mortality [4,5]. The mechanism(s) underlying this severe systemic inflammation is unknown. The use of classical anti-inflammatory agents, such as anti-tumour 


\section{JOURNAL OF HEPATOLOGY}

necrosis factor or steroids in ACLF is fraught with potential difficulties as infection and immune failure are also important features of this syndrome and when present, is associated with a high risk of mortality [2,5-7]. Treatment of ACLF is an unmet need and modulation of inflammatory response in ACLF is an important potential target of therapy [8].

Recently, much controversy has arisen in the literature due to the suggestion that the use of non-selective beta blockers (NSBBs) in patients with refractory ascites $[9,10]$, spontaneous bacterial peritonitis [11] and alcoholic hepatitis [12], may increase mortality rates through accentuation of circulatory disturbances and the resultant renal failure. These studies are limited in their design as they address specific subgroups of patients such as refractory ascites and spontaneous bacterial peritonitis and lack prospective data assessing extrahepatic, extrarenal organ functions as well as overall severity using prognostic scores other than liver-specific scores. Despite these limitations, many clinicians are ceasing to use this potentially lifesaving drug in patients with advanced cirrhosis despite another study suggesting improved outcomes for patients on the waiting list for liver transplantation [13].

NSBBs have been studied extensively in cirrhotic patients and are the drug of first choice for primary and secondary prophylaxis of variceal bleeding, as it has been incontrovertibly shown to have beneficial effects on the severity of portal hypertension [14]. These effects require both the beta- 1 and beta- 2 actions of the drug to ameliorate splanchnic vasodilation and high cardiac output [15]. NSBBs have many other potential beneficial actions in patients with cirrhosis through its action on increasing gut motility and reducing bacterial translocation, which would reduce systemic inflammation and therefore have beneficial effects in ACLF patients over and above its hemodynamic effects $[16,17]$.

This study was designed to test the hypothesis that cirrhotic patients being treated with NSBBs would have reduced systemic inflammation and reduced mortality if they developed ACLF. In order to test this, we used the data from the CANONIC study, which is a prospective, observational study performed in 1349 cirrhotic patients included from 29 European hospitals [2]. The present analysis is focused on the ACLF cohort. The data from this study was previously used to derive the diagnostic and prognostic criteria for ACLF, which were validated in independent cohorts [2-4]. The specific aims of this study were to evaluate the clinical effects of ongoing administration of NSBBs in hospitalized cirrhotic patients who developed ACLF, focusing on safety of its use, effects on organ function and mortality, clinical course of ACLF and effects on inflammatory markers.

Table 1. Characteristics at ACLF diagnosis in patients receiving and not receiving NSBBs within the previous 3 months.

\begin{tabular}{|c|c|c|c|}
\hline Characteristics & $\begin{array}{l}\text { No NSBB } \\
\mathrm{N}=185\end{array}$ & $\begin{array}{l}\text { Use of } \\
\text { NSBBs } \\
N=164\end{array}$ & $p$ value \\
\hline Age (yr) & $53.6(11.5)$ & $58.1(11.8)$ & 0.0003 \\
\hline Male sex & $117(63.2 \%)$ & $111(67.7 \%)$ & 0.3844 \\
\hline \multicolumn{4}{|l|}{ Cause of cirrhosis: } \\
\hline Alcohol alone & $113(62.4 \%)$ & $85(54.1 \%)$ & 0.1228 \\
\hline $\mathrm{HCV}$ alone & $22(12.2 \%)$ & $26(16.6 \%)$ & 0.2471 \\
\hline $\mathrm{HCV}+$ alcohol & $20(11.1 \%)$ & $11(7.0 \%)$ & 0.1990 \\
\hline Previous decompensations & $116(65.5 \%)$ & $137(86.7 \%)$ & $<0.0001$ \\
\hline Ascites & $102(87.9 \%)$ & $118(86.8 \%)$ & 0.7817 \\
\hline Hepatic encephalopathy & $56(49.1 \%)$ & $66(49.6 \%)$ & 0.9374 \\
\hline Gastrointestinal bleeding & $19(16.8 \%)$ & $58(43.3 \%)$ & $<0.0001$ \\
\hline Spontaneous bacterial peritonitis & $19(17.3 \%)$ & $20(15.5 \%)$ & 0.7122 \\
\hline Other & $23(20.7 \%)$ & $26(19.9 \%)$ & 0.8662 \\
\hline \multicolumn{4}{|l|}{ Potential precipitating events of ACLF: } \\
\hline At least one PE & $110(62.2 \%)$ & $95(59.8 \%)$ & 0.6527 \\
\hline$>1 \mathrm{PE}$ & $41(23.2 \%)$ & $26(16.4 \%)$ & 0.1187 \\
\hline Bacterial infection & $57(30.8 \%)$ & $53(32.3 \%)$ & 0.7624 \\
\hline Gastrointestinal hemorrhage & $31(16.8 \%)$ & $22(13.4 \%)$ & 0.3852 \\
\hline $\begin{array}{l}\text { Active alcoholism before } \\
\text { admission }\end{array}$ & $50(28.9 \%)$ & $28(18.1 \%)$ & 0.0214 \\
\hline Other PEs & $13(7.3 \%)$ & $13(8.3 \%)$ & 0.7388 \\
\hline Ascites clinically diagnosed & $149(81.0 \%)$ & $119(73.0 \%)$ & 0.0771 \\
\hline Ascites + subrogates of ascites & $177(95.7 \%)$ & $158(96.3 \%)$ & 0.7517 \\
\hline Mean arterial pressure $(\mathrm{mmHg})$ & $78.9(12.7)$ & $78.2(13.3)$ & 0.6639 \\
\hline Heart rate $(\mathrm{bpm})$ & $89.8(19.0)$ & $79.0(19.5)$ & $<0.0001$ \\
\hline \multicolumn{4}{|l|}{ Organ failures: } \\
\hline Liver & $80(43.2 \%)$ & $57(34.8 \%)$ & 0.1051 \\
\hline Kidney & $89(48.1 \%)$ & $89(54.3 \%)$ & 0.2506 \\
\hline Cerebral & $51(27.6 \%)$ & $30(18.3 \%)$ & 0.0405 \\
\hline
\end{tabular}

\begin{tabular}{llll}
\hline Characteristics & No NSBB & Use of & $p$ value \\
& $\mathrm{N}=185$ & $\mathrm{NSBBs}$ & \\
& & $\mathrm{N}=164$ & \\
\hline Coagulation & $66(35.7 \%)$ & $42(25.6 \%)$ & 0.0423 \\
Circulation & $35(18.9 \%)$ & $27(16.5 \%)$ & 0.5492 \\
Lungs & $19(10.3 \%)$ & $19(11.6 \%)$ & 0.6938 \\
Kidney dysfunction & $35(22.2 \%)$ & $19(13.8 \%)$ & 0.0624 \\
Mild to moderate hepatic & $54(34.2 \%)$ & $51(37.0 \%)$ & 0.6281 \\
encephalopathy & & & \\
\hline CLIF-C ACLF score & $51.4(10.2)$ & $49.5(10.0)$ & 0.1468 \\
MELD score & $28.9(7.4)$ & $27.1(7.6)$ & 0.0546 \\
\hline Laboratory data: & & & \\
Hematocrit (\%) & $27(5)$ & $27(5)$ & 0.6888 \\
Platelet count (x10\%/L) & $97(77)$ & $81(60)$ & 0.0492 \\
Serum bilirubin (mg/dl) & $13.8(11.9)$ & $10.1(11.0)$ & 0.0072 \\
International normalized ratio & $2.3(1.0)$ & $2.2(1.0)$ & 0.3580 \\
Alanine aminotransferase (U/L) & $35(22-66)$ & $34(21-66)$ & 0.5149 \\
Aspartate aminotransferase (U/L) & $77(41-143)$ & $68(35-123)$ & 0.4698 \\
Y-Glutamyltransferase (U/L) & $77(30-151)$ & $70(36-138)$ & 0.6451 \\
Serum creatinine (mg/dl) & $2.0(1.4)$ & $2.1(1.3)$ & 0.4328 \\
Serum sodium (mmol/L) & $134.3(6.7)$ & $136.0(6.1)$ & 0.0199 \\
WBC (x10 $/ L)$ & $10.8(6.6)$ & $8.5(5.8)$ & 0.0021 \\
Plasma C-reactive protein (mg/L) & $33.5(16-54)$ & $25.4(13-52)$ & 0.4664 \\
\hline ACLF grade: & & & \\
ACLF-1 & $81(43.8 \%)$ & $91(55.5 \%)$ & \\
ACLF-2 & $73(39.5 \%)$ & $57(34.8 \%)$ & \\
ACLF-3 & $31(16.7 \%)$ & $16(9.7 \%)$ & 0.0474 \\
\hline Liver transplantation after 28 days & $15(8.1 \%)$ & $17(10.4 \%)$ & 0.2009 \\
Liver transplantation after 90 days & $24(13.1 \%)$ & $26(16.2)$ & 0.4660 \\
28-day mortality & $63(34.1 \%)$ & $40(24.4 \%)$ & 0.0482 \\
90-day mortality & $83(44.9 \%)$ & $63(38.4 \%)$ & 0.2228 \\
\hline & & & \\
\hline & & & \\
\hline
\end{tabular}

Data are $\mathrm{n}(\%)$, mean (standard deviation) or median (Inter-quartile range) NSBBs, non-selective beta blockers. 


\section{Research Article}

\section{Patients and methods}

Patients

The study was performed in 349 patients with ACLF and complete set of data included in the CANONIC study, which is a prospective observational investigation in 1349 patients with cirrhosis (out of 2149 screened) who were admitted to 29-European hospitals within a period of 6 months for the treatment of an acute decompensation (AD) (ascites, encephalopathy, gastrointestinal hemorrhage and/or bacterial infections) [2]. Among the causes of exclusion the most frequent were: hospitalization for a scheduled diagnostic or therapeutic procedure hepatocellular carcinoma outside the Milan Criteria, patient's refusal to participate and presence of severe chronic extrahepatic disease. Two hundred and seventy-seven patients presented with ACLF at study enrollment and 72 (20.6\%) developed ACLF during hospitalization.

Data collection

Data from history, including events that may be potential precipitating factors of ACLF, physical examination and laboratory measurements were obtained at ACLF diagnosis in all patients as described previously [2]. Special attention was given to data from the previous 3-months up to ACLF development, including alcohol drinking habit of the patients, therapeutic procedures (paracentesis with and without albumin, transjugular intrahepatic portosystemic shunting, major surgery) and pharmacological treatments (primary or secondary prophylaxis of bacterial infections and gastrointestinal hemorrhage with norfloxacin, secondary prophylaxis of hepatic encephalopathy with lactulose or rifaximin and treatment with diuretics or antibiotics). Following diagnosis of ACLF, an intensive prespecified 28-day follow-up data collection was performed in all patients. Finally as pre-specified in the study protocol, information on liver transplantation, mortality and causes of death during 1 -year were obtained for all study patients. Data were collected using an electronic case report-form. The main end-point for the present study focused on 28-day survival.

Data about use of NSBBs

The data about the use of NSBBs, its type and dosage was specifically recorded in the case notes. If the patients were not receiving NSBBs at the time of hospital admission, a specific note about when they were started on therapy was noted.

Diagnostic criteria

Diagnostic criteria of organ failure were based on the CLIF-C organ failure score [4]: Liver Failure: serum bilirubin $\geqslant 12 \mathrm{mg} / \mathrm{dl}$; Renal failure: serum creatinine $\geqslant 2 \mathrm{mg} / \mathrm{dl}$; Cerebral failure: grade III-IV hepatic encephalopathy (West-Haven classification); Coagulation failure: international normalized ratio (INR) $\geqslant 2.5$; Circulatory failure: use of vasoconstrictors to treat severe arterial hypotension (use of vasoconstriction for the treatment of type $1 \mathrm{HRS}$ in patients without severe hypotension not included); Respiratory failure: $\mathrm{PaO}_{2} / \mathrm{FiO}_{2} \geqslant 200$ or $\mathrm{SpO}_{2} / \mathrm{FiO}_{2}$ $\geqslant 214$. Renal dysfunction was diagnosed when serum creatinine ranged between 1.5 and $1.9 \mathrm{mg} / \mathrm{dl}$; cerebral dysfunction was diagnosed in patients with Grade I or Grade II hepatic encephalopathy. Type 1 ACLF defines the presence of renal failure alone or of any other type of single renal failure if associated to renal dysfunction and/or cerebral dysfunction. Type II ACLF and type III ACLF define the presence of 2 and 3 to 6 organ failures, respectively.

Statistical analysis

Data were summarized by means of the appropriate descriptive statistics: means and Standard Deviation (SD) or median and Inter-quartile range (IQR) values for continuous variables, frequencies and percentages for categorical parameters. Univariate analyses of the effect of the treatment with NSBBs included student's $t$ test or Mann-Whitney $U$ test for parametric or non-parametric pair-wise comparisons, respectively, and Chi-square tests for categorical variables. Survival curves were estimated by means of Kaplan-Meier method and compared using the log-rank test.

The main study objective was that of assessing the effects of the administration of NSBBs on 28-day mortality in ACLF patients. Patients' characteristics prior to ACLF onset (corresponding to study inclusion for most patients) significantly associated with both the treatment and mortality were taken into account as potential confounders to adjust treatment effects. A logistic regression model was fitted to adjust the effect of NSBBs for the potential confounders, which were kept in the final model only if they led at least to a $10 \%$ change in the model coefficient estimated for the use of NSBBs. A logistic regression model was also fitted to estimate the probability of death at 28 days in patients taking or not taking NSBBs at different levels of severity of the patients, as measured by the CLIF-C ACLF score. In all statistical comparisons, a 0.05 significance level (two-tailed) was assumed.

\section{Results}

Differences in clinical and laboratory characteristics between patients with ACLF who did and did not received NSBBs prior to enrollment

Of the 349 patients with valid data on NSBB use and developing ACLF at enrollment or during the hospitalization, 164 (47\%) received NSBBs. Of these, 9 patients were started on therapy with NSBBs after hospital admission whereas 155 received NSBB within 3 months prior to ACLF diagnosis. One hundred and eighty-five patients did not receive NSBBs. Similar numbers of patients in the two groups received antibiotics within 3-months of hospital admission (NSBB: 45.7\%; No NSBB: 41.6\%; $p=0.43$ ). One hundred and eleven patients $(67.7 \%)$ received propranolol at a median dose of $40 \mathrm{mg}(20-80)$ per day, 6 patients were taking nadolol (3.7\%; $40 \mathrm{mg}(40-80)$ ), 16 were taking carvedilol (10\%; $12.5 \mathrm{mg}(6.25-25))$ and 31 were taking 'other' NSBBs (18.9\%; $40 \mathrm{mg}$ (25-80)). Table 1 shows the clinical, laboratory, liver transplantation and mortality data in these patients. There were no significant differences between patient characteristics dependent on when the NSBBs were started (Supplementary Table 1). Also, neither the type of NSBB administered nor its dose of NSBBs was associated with mortality or effect on heart rate and white cell count (Supplementary Tables 2 and 3). Therefore, further analyses were performed to determine the differences between NSBB administered group $(\mathrm{n}=164)$ vs. those not administered NSBBs $(\mathrm{n}=185)$.

Of the patients in the NSBB administered group, 77 patients continued NSBBs following the diagnosis of ACLF, but the dose was reduced in 8 patients. In 78 patients, the NSBBs were stopped prior to inclusion in the study. In 68 of the 78 patients who discontinued NSBB after study enrollment (87\%) ACLF was diagnosed at study inclusion. The rate of ACLF development after study inclusion was similar in both groups $(13 \%$ in patients discontinuing the treatment vs. $17 \%$ in patients continuing with NSBB) (Supplementary Table 4).

Patients receiving NSBBs were significantly older and had significantly higher prevalence of prior episodes of previous decompensation than patients not receiving NSBBs. This latter finding was due to a significantly higher prevalence of prior episodes of gastrointestinal hemorrhage in patients receiving NSBBs. The remaining previous decompensating events were similar in the two groups. There were no differences between groups in the etiology of cirrhosis, number of patients with precipitating events and its type except for active alcoholism within the 3-months prior to hospitalization, which was significantly more prevalent in patients not receiving NSBBs. There was also no difference between groups in relation to the prevalence of ascites, either as assessed by the presence of clinically detectable ascites at ACLF diagnosis or by the sum of clinically detectable ascites at enrollment and surrogates of ascites (treatment with diuretics or paracentesis or spontaneous bacterial peritonitis) within 1-week prior of after diagnosis. 
As expected, heart rate was significantly lower in patients receiving NSBBs. This was associated with a higher serum sodium concentration and, white cell count and bilirubin were significantly lower in patients receiving NSBBs. MELD score was lower in the patients treated with NSBBs which did not reach statistical significance (28.9 (7.4) vs. 27.1 (7.6); $p=0.056$ ). There were no significant differences between groups in mean arterial pressure, hematocrit, serum creatinine and plasma C-reactive protein.

Organ failures, prognostic scores and ACLF grades at diagnosis and course of ACLF during 3-7 days after diagnosis

The prevalence of cerebral failure and coagulation failure was significantly lower in patients receiving NSBBs. The prevalence of other organ failures between groups was not significantly different. There were no significant differences in cerebral dysfunction but renal dysfunction was lower in the patients treated with NSBBs $(p=0.06)$. Creatinine levels at last study visit were similar between study groups. (NSBB: $1.79 \mathrm{mg} / \mathrm{dl}$ (1.39); No NSBB: $1.73 \mathrm{mg} / \mathrm{dl}(1.12) . p=0.67)$. The CLIF-C ACLF scores were similar between the groups (Table 1 ). There were, however significant differences in the severity of ACLF. The prevalence of ACLF- 1 was higher in patients receiving NSBBs. In contrast, the prevalence of ACLF-2 and ACLF-3 was higher in patients not receiving NSBBs.

The early clinical course of ACLF was significantly worse in patients not receiving NSBBs. The evolution of ACLF grade after 3-7 days from the diagnosis in the 319 patients with complete data is shown in Table 2. Among patients not taking NSBBs, 40 (23.4\%) presented a clinical course to more severe grade of the syndrome, in comparison with 21 patients $(14.2 \%)$ in the NSBBtreatment group ( $p=0.0371$ ). This suggests a possible protective effect of the treatment with NSBBs against the progression of the syndrome (Relative Risk: 0.61; 95\%CI: 0.38-0.98). Moreover, the number of patients presenting a complete resolution of the initial ACLF was higher in the NSBB treated group compared with the untreated patients (46.6\% vs. $37.4 \%$ ), although in this case statistical significance was not reached $(p=0.0967)$. Fig. 1 shows the improvement or worsening in ACLF severity observed after 3-7 days from diagnosis. Significantly, more patients in the NSBB treated group presented a 1-grade reduction in ACLF classification $(43.2 \% v s$. $27.5 \%, p=0.0032)$, while 1 -grade worsening was significantly higher in patients not treated with NSBBs $(18.1 \%$ vs. $10.1 \%, p=0.0427$ ).

\section{JOURNAL OF HEPATOLOGY}

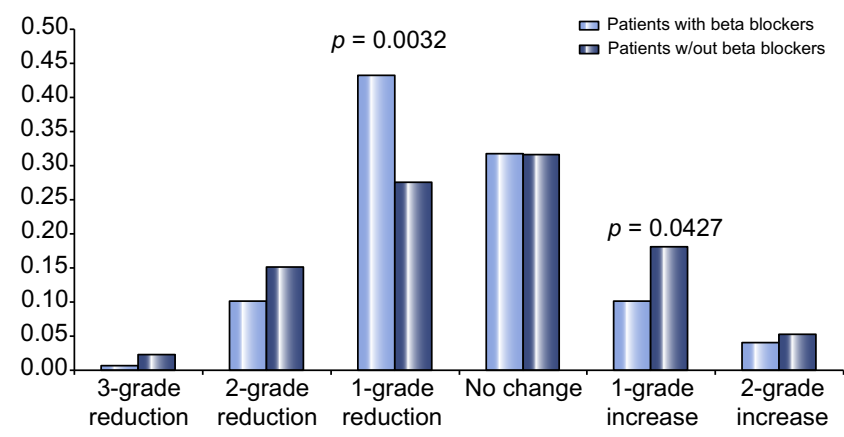

Fig. 1. Evolution of the ACLF grade one week after diagnosis by use of nonselective beta blockers.

\section{Mortality and survival probability}

Short-term (28-day) mortality rate (Table 1) was significantly higher and the probability of survival (Fig. 2) significantly lower in patients not receiving NSBBs. When the probability of death for patients with and without NSBBs was estimated for the different values of the CLIF-C ACLF score (Fig. 3), the curve obtained in patients receiving NSBBs was significantly shifted to the right, indicating that for identical values of the score mortality was lower in treated patients. Six-month and 1-year mortality rates were similar between the groups (NSBB: $47.6 \%$ and $51.8 \%$; No NSBB: $49.7 \%$ and $56.8 \% ; p=0.64$ and 0.35 respectively).

\section{Effect of the treatment with NSBBs on survival}

Table 3 shows an analysis of potential risk factors of mortality in the univariate analysis. A history of more than one precipitating event, higher heart rate, higher prevalence of organ failure (statistically significant only for liver, cerebral and circulation failure), higher CLIF-C ACLF score and MELD score, higher serum bilirubin, INR, aspartate aminotransferase, serum creatinine and white cell count (WCC), higher grade of ACLF and no treatment with NSBBs were significantly associated with short-term mortality rate.

None of the baseline patients' characteristics (age, gender, etiology of the cirrhosis, previous decompensations, events potentially precipitating the ACLF or clinical assessments) were found to be significantly associated with treatment with NSBBs

Table 2. Evolution of ACLF grade up to one week after its first onset according to treatment with NSBBs.

\begin{tabular}{|c|c|c|c|c|c|c|c|c|c|}
\hline \multirow{3}{*}{$\begin{array}{l}\text { ACLF grade at the } \\
\text { 1st onset }\end{array}$} & \multicolumn{4}{|c|}{ ACLF grade after 3-7 days from the onset } & ACLF grade at & \multicolumn{4}{|c|}{ ACLF grade after 3-7 days from the onset } \\
\hline & \multicolumn{4}{|c|}{ No NSBBs $(n=171)$} & & \multicolumn{4}{|c|}{ Use of NSBBs $(n=148)$} \\
\hline & NO ACLF & ACLF-1 & ACLF-2 & ACLF-3 & & NO ACLF & ACLF-1 & ACLF-2 & ACLF-3 \\
\hline ACLF-1 $(n=76)$ & $36(47.4 \%)$ & $19(25.0 \%)$ & $12(15.8 \%)$ & $9(11.8 \%)$ & ACLF-1 $(n=83)$ & $53(63.9 \%)$ & $19(22.9 \%)$ & $5(6.0 \%)$ & $6(7.2 \%)$ \\
\hline ACLF-2 $(n=68)$ & $24(35.3 \%)$ & $7(10.3 \%)$ & $18(26.5 \%)$ & $19(27.9 \%)$ & ACLF-2 $(n=52)$ & $15(28.9 \%)$ & $9(17.3 \%)$ & $18(34.6 \%)$ & $10(19.2 \%)$ \\
\hline ACLF-3 $(n=27)$ & $4(14.8 \%)$ & $2(7.4 \%)$ & $4(14.8 \%)$ & $17(63.0 \%)$ & ACLF-3 $(n=13)$ & $1(7.7 \%)$ & 0 & $2(15.4 \%)$ & $10(76.9 \%)$ \\
\hline \multicolumn{10}{|c|}{ Evolution of the initial ACLF grade } \\
\hline \multicolumn{2}{|c|}{ Resolution of the ACLF } & \multicolumn{3}{|c|}{$64 / 171(37.4 \%)$} & \multicolumn{2}{|c|}{ Resolution of the ACLF } & \multicolumn{2}{|c|}{$69 / 148(46.6 \%)$} & $p=0.0967$ \\
\hline \multicolumn{2}{|c|}{ Worsening of the ACLF } & \multicolumn{3}{|c|}{$40 / 171(23.4 \%)$} & \multicolumn{2}{|c|}{ Worsening of the ACLF } & \multicolumn{2}{|c|}{$21 / 148(14.2 \%)$} & $p=0.0371$ \\
\hline
\end{tabular}

NSBBs, non-selective beta blockers. 


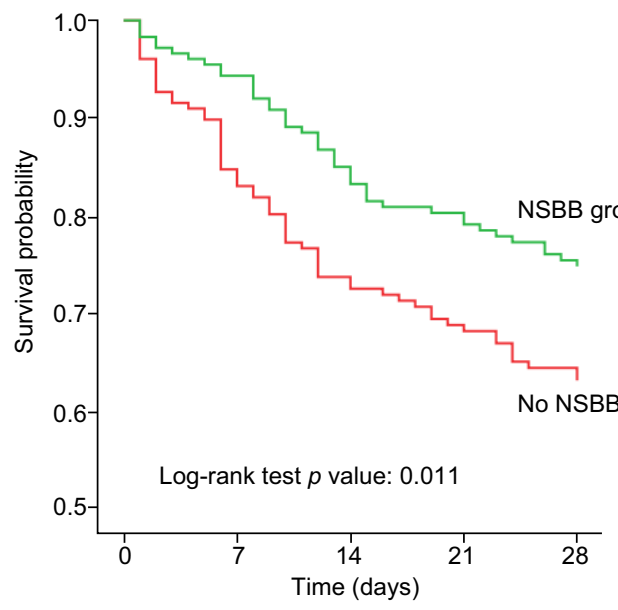

Fig. 2. Kaplan-Meier survival curves at 28 days after diagnosis in ACLF patients taking (green line) and not taking (red line) non-selective beta blockers. (This figure appears in colour on the web.)

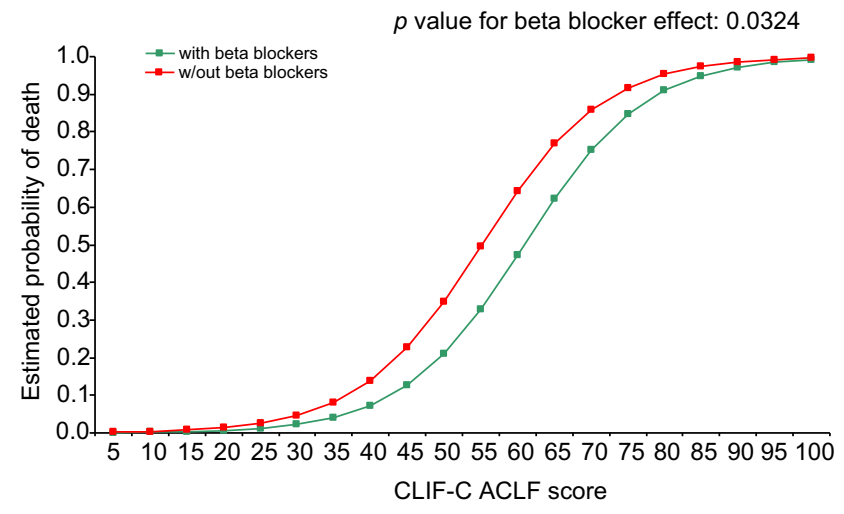

Fig. 3. Estimated probability of death at 28 days from logistic regression model in ACLF patients with (green line) or without (red line) non-selective beta blockers by CLIF-C ACLF score levels. (This figure appears in colour on the web.)

and mortality (Tables 1 and 3). In order to adjust treatment effects for the observed imbalances in the factors unrelated to the severity of liver disease at hospital admission, a logistic regression model for 28-day mortality was fitted including age, presence of previous decompensations and active alcoholism within the 3 months prior to hospitalization together with the treatment. The adjusted protective effect of the treatment estimated through this model was statistically significant. The estimated risk-reduction (OR) associated to the use of NSBBs was 0.596 (95\% CI: $0.361-0.985 ; p=0.0436$ ). One death at 28 days could be prevented for every 110.097 (95\% CI: 6-334) patients that are treated with NSBBs. The main causes of death were similar in both groups and due predominantly to progressive multiorgan failure or septic shock (Supplementary Table 5).

28-day (37.2\% vs. $13 \%$; $p<0.0005)$ and 3-month mortality ( $50 \%$ vs. $14.7 \% ; p<0.001$ ) were significantly higher in the patients that stopped NSBBs after development of ACLF compared with those that continued to take NSBBs. Although the MELD scores in these groups were similar, the patients that stopped NSBBs were more likely to have circulatory and lung failure and higher CLIF-C ACLF scores (51.6 (11.3) vs. 46.9 (8.2); $p<0.02$ ). Mean arterial pressure was similar between the groups but the heart rate was lower in the group that continued NSBBs. Inflammatory markers, WCC and C-reactive protein (CRP) were similar (Supplementary Table 4).

Relationship between the use of NSBBs, white cell count and severity of ACLF

Table 4 shows the relationship between the use of NSBBs, white cell count and the severity of ACLF. For ACLF grades 1 and 2 at presentation, the WCC was significantly lower in the patients treated with NSBBs compared with the untreated patients but the counts were similar in the ACLF grade 3 patients (Table 4A). In the patients that worsened their ACLF grade, the WCC was also significantly higher in the patients not being treated with NSBBs compared with the patients treated with NSBBs (Table 4B).

\section{Discussion}

The results of this study show for the first time that in patients with cirrhosis presenting with ACLF, NSBBs were safe to administer and its use was independently associated with improved 28-day survival. This reduction in short-term mortality with the use of NSBBs was due to significantly greater number of patients having lower grades of ACLF at presentation and, in those with ACLF, resolving ACLF completely or to lower grades. This reduction in 28-day mortality did not translate into improved long-term mortality and this may be due to the relatively fewer additional deaths at the 6-month and 1-year time points. The mechanism of this improvement may be an NSBB-induced reduction in the severity of systemic inflammatory response indicated by lower WCC and CRP both at the time of presentation and also during hospital follow-up. The results suggest that ongoing treatment with NSBBs in cirrhotic patients reduces the severity of subsequent ACLF and prevents its progression to more advanced stages, which together contributes to lower mortality.

Systemic inflammation, characterized by an increased WCC and heart rate are the pathophysiological hallmarks of ACLF $[1,2,4,5,8]$. As shown in previous studies, these markers were significantly more elevated in the ACLF patients who died and the WCC was an independent predictor of mortality [2,4]. The administration of NSBBs was associated with a significant reduction in WCC, which was consistent with a reduction in the heart rate. As illustrated in Table 4, in patients not treated with NSBBs, the WCC was higher in the matched patients with ACLF grades 1 and 2. It is notable that the WCC was also significantly higher in the patients who progressed to the more advanced stages of the disease. The exact mechanism of how this is achieved is not clear and may indicate an effect on the sympathetic nervous system, which is known to contribute to immune cell failure in sepsis [19-22]. This hypothesis will need to be explored in future studies. Additionally, NSBBs may reduce systemic inflammation through its action on increasing gut motility and reducing bacterial translocation $[16,17]$, which is known to sensitize end organs to a subsequent inflammatory injury [23]. This thinking is the rationale behind the concept of gut sterilization using Norfloxacin and Rifaximin, which have been shown to reduce the incidence of renal failure and hepatic encephalopathy in susceptible patients $[24,25]$. In keeping with this hypothesis, a recent meta-analysis showed that NSBBs reduced the risk of 


\section{JOURNAL OF HEPATOLOGY}

development of spontaneous bacterial peritonitis (SBP) in susceptible cirrhotic patients [26]. Alternatively, the observed effect of NSBBs may represent a direct drug effect. Esmolol, a selective beta-1 blocker was studied in patients with severe septic shock (without underlying cirrhosis) and shown to be safe with potentially beneficial effects on multi-organ function and inflammatory markers [18]. Taken together, many interacting mechanisms working through both the beta- 1 and the beta- 2 pathways may be involved in the modulation of systemic inflammation observed in the present study. Further studies will be needed to look at the potential role of adrenoreceptors, severity of activation of the sympathetic nervous system and their role in modulation of inflammatory responses.

NSBBs were first reported to have adverse survival outcomes in patients with refractory ascites in retrospective studies [9]. The proposed mechanism underlying this increased mortality of patients with refractory ascites was a greater incidence of postparacentesis circulatory dysfunction in the patients being treated with NSBBs [10]. In the present study, similar numbers of patients in either group had 'severe ascites' and renal failure. Mean arterial pressure in the two cohorts was also similar at presentation. More recent studies evaluating larger numbers of patients have not confirmed the observation of increased mortality in patients with refractory ascites $[11,13]$. In fact, in a multicenter study in decompensated patients on the waiting list for liver transplantation, administration of NSBBs was associated with better survival [13]. Furthermore, in a recent systematic review of 9 trials of NSBBs, 1 case-control study and 4 retrospective analyses, increased mortality was only observed in 1 study described above [27]. In keeping with these latter studies, the data from the present, study has not found any evidence that patients with mild or severe ascites with or without its surrogates do any worse with NSBBs.

In the past 5-years or so, data from retrospective studies have questioned the role of NSBBs in patients with SBP [11]. Bacterial infection as a cause of acute decompensation in the present study was similar in the patients treated with or without NSBBs, which is in keeping with data from the US showing no differences in the rates of infection in compensated or decompensated cirrhotic patients [28]. Importantly, survival in the present study was not related to the presence, type or site of infection. This observation is important given the recent debate about the use of NSBBs in patients with SBP $[11,13,16]$. In a large single center retrospective study from Austria, which included 182 patients, the investigators suggested that the administration of NSBBs to patients with SBP is associated with greater risk of renal failure and death [11]. In the SBP sub-cohort in the present study, there was no difference in the mortality of patients treated with NSBB and those without. Serste et al. recently reported that the use of NSBBs were associated with increased risk of acute kidney injury in patients with alcoholic hepatitis, which was also not confirmed in the present study [12]. These data argue strongly against stopping NSBBs in patients with refractory ascites, SBP or alcoholic hepatitis unless there are specific contraindications.

It is important to note that the dose of NSBBs used in the present study, in most of the patients was $40 \mathrm{mg}$ (20-80) propranolol per day or equivalent, which is lower than in most of the previous studies described above [9-12]. The effect of the NSBB in ACLF seems to be class related as no differences in outcomes were observed with propranolol or other NSBBs. A further analysis of the patients receiving low doses, i.e. $<40 \mathrm{mg}$ compared with those on doses of $40 \mathrm{mg}$ or more did not show any significant differences between the groups in terms of effect on inflammatory response or mortality. Paradoxically, the mean arterial pressure was higher in those treated with the higher dose but the heart rates were similar indicating that these higher doses were not deleterious if they did not adversely affect mean arterial pressure. The exact mechanism underlying the beneficial effect of NSBBs at this low dose is unclear and further studies will be required to determine whether the non-hemodynamic beneficial effects of NSBBs in advanced cirrhotic patients can be achieved at these lower doses. Despite the occurrence of ACLF, about $50 \%$ patients were continued on therapy with NSBBs, the dose of which was reduced in a small proportion of patients ( 8 patients; $10.3 \%$ ). The 28-day and 90-day mortality of the patients who continued on therapy with NSBBs was significantly lower than the patients in whom NSBBs were withdrawn. This effect was independent of any significant differences in the markers of systemic inflammation such as WCC or CRP. Although the heart rate was lower in the patients who continued on therapy with NSBBs, mean arterial pressure was similar. It is not possible to conclude that the improved survival was due to a drug effect of continued NSBB use as the patients in whom NSBBs were stopped had higher number of patients with ACLF3, circulatory and lung failure resulting in higher CLIF-C ACLF score. Specifically designed studies need to be performed to answer this question.

Patients on NSBBs tended to be older with a history of previous decompensation and were less likely to be alcoholics who were drinking actively. These were associated with a significantly lower heart rate and WCC. In the present study, which was focused on ACLF patients, alcoholism was not associated with increased 28-day mortality. The reason behind the prescribing differences of NSBBs in the present study can perhaps be explained by the fact that patients actively abusing alcohol are more likely to present for the first time with $A D$ and patients with previous decompensation have had the opportunity to have been started on NSBBs during their previous decompensation. However, why some patients were selected for treatment with NSBBs is not clear from the CANONIC database. NSBBs were more likely to be prescribed in patients who had a previous variceal bleed, which is a group with higher potential risk of death compared with a population without a previous variceal bleed. Mortality from variceal bleeding was similar in the cohorts treated with or without NSBBs. The protection observed in these patients from the effects of NSBBs is therefore even more noteworthy [28]. In order to account for these variables a logistic regression model was developed and the improvement in survival with the use of NSBBs was independent of these variables.

The occurrence of ACLF in the patients treated with NSBBs compared with those without was similar but the number of organ failures reflecting the severity of ACLF was lower in the NSBB treated patients. In fact, as illustrated in Tables 1 and 2, the administration of NSBBs was an independent predictor of a greater likelihood of the patients having ACLF-1 compared with ACLF-3, which is associated with significantly worse prognosis $[2,4]$. As illustrated in Fig. 3, and shown previously, it is clear that the 28-day mortality of patients with ACLF is predicted by the CLIF-C ACLF score, which is a combination of the CLIF-C organ failure score, WCC and age [4]. Fig. 3 reveals that for any given CLIF-C ACLF score (except at the 2 extreme ends where the mortality rates are very low or very high), the mortality of patients treated with NSBBs was significantly lower indicating further 


\section{Research Article}

\begin{tabular}{|c|c|c|c|}
\hline Characteristics & $\begin{array}{l}\text { 28-day survivors } \\
\mathrm{N}=246\end{array}$ & $\begin{array}{l}\text { 28-day deaths } \\
\mathrm{N}=103\end{array}$ & $p$ value \\
\hline Age (yr) & $55.5(12.3)$ & $56.2(10.6)$ & 0.6222 \\
\hline Male sex & $168(68.3 \%)$ & $60(58.3 \%)$ & 0.0722 \\
\hline \multicolumn{4}{|l|}{ Cause of cirrhosis: } \\
\hline Alcohol alone & $135(56.5 \%)$ & $63(63.6 \%)$ & 0.2245 \\
\hline HCV alone & $33(13.8 \%)$ & $15(15.2 \%)$ & 0.7473 \\
\hline $\mathrm{HCV}+$ alcohol & $26(10.9 \%)$ & $5(5.1 \%)$ & 0.1010 \\
\hline Other causes & $45(18.8 \%)$ & $16(16.2 \%)$ & 0.5618 \\
\hline Previous decompensation & $183(77.9 \%)$ & $70(70.0 \%)$ & 0.1251 \\
\hline Ascites & $160(87.9 \%)$ & $60(85.7 \%)$ & 0.6388 \\
\hline Hepatic encephalopathy & $84(46.9 \%)$ & $38(55.9 \%)$ & 0.2086 \\
\hline Gastrointestinal bleeding & $53(29.4 \%)$ & $24(35.8 \%)$ & 0.3361 \\
\hline $\begin{array}{l}\text { Spontaneous bacterial } \\
\text { peritonitis }\end{array}$ & $31(17.8 \%)$ & $8(12.3 \%)$ & 0.3052 \\
\hline Other & $39(22.3 \%)$ & $10(14.9 \%)$ & 0.2023 \\
\hline \multicolumn{4}{|c|}{ Potential precipitating events of ACLF: } \\
\hline At least one PE & $140(59.6 \%)$ & $65(64.4 \%)$ & 0.4099 \\
\hline$>1 \mathrm{PE}$ & $39(16.6 \%)$ & $28(27.7 \%)$ & 0.0193 \\
\hline Bacterial infection & $73(29.7 \%)$ & $37(35.9 \%)$ & 0.2519 \\
\hline $\begin{array}{l}\text { Gastrointestinal } \\
\text { hemorrhage }\end{array}$ & $32(13.0 \%)$ & $21(20.4 \%)$ & 0.0797 \\
\hline $\begin{array}{l}\text { Active alcoholism before } \\
\text { admission }\end{array}$ & $53(23.1 \%)$ & $25(25.3 \%)$ & 0.6805 \\
\hline Other PE's & $16(6.8 \%)$ & $10(9.9 \%)$ & 0.3362 \\
\hline Ascites clinically diagnosed & $185(75.8 \%)$ & $83(80.6 \%)$ & 0.3337 \\
\hline $\begin{array}{l}\text { Ascites + subrogates of } \\
\text { ascites }\end{array}$ & $235(95.6 \%)$ & $100(97.1 \%)$ & 0.7655 \\
\hline $\begin{array}{l}\text { Mean arterial pressure } \\
(\mathrm{mmHg})\end{array}$ & 79.5 (11.9) & $76.6(14.9)$ & 0.1094 \\
\hline Heart rate $(\mathrm{bpm})$ & $81.7(18.8)$ & $91.4(20.8)$ & $<0.0001$ \\
\hline
\end{tabular}

\begin{tabular}{|c|c|c|c|}
\hline Characteristics & $\begin{array}{l}\text { 28-day survivors } \\
\mathrm{N}=246\end{array}$ & $\begin{array}{l}\text { 28-day deaths } \\
\mathrm{N}=103\end{array}$ & $p$ value \\
\hline \multicolumn{4}{|l|}{ Organ failures: } \\
\hline Liver & $86(35.0 \%)$ & $51(49.5 \%)$ & 0.0111 \\
\hline Kidney & $119(48.4 \%)$ & $59(57.3 \%)$ & 0.1289 \\
\hline Cerebral & $47(19.1 \%)$ & $34(33.0 \%)$ & 0.0050 \\
\hline Coagulation & $70(28.5 \%)$ & $38(36.9 \%)$ & 0.1199 \\
\hline Circulation & $35(14.2 \%)$ & $27(26.2 \%)$ & 0.0075 \\
\hline Lungs & $23(9.4 \%)$ & $15(14.6 \%)$ & 0.1538 \\
\hline Kidney dysfunction & $34(16.8 \%)$ & $20(21.3 \%)$ & 0.2173 \\
\hline $\begin{array}{l}\text { Mild to moderate hepatic } \\
\text { encephalopathy }\end{array}$ & $71(35.2 \%)$ & $34(36.2 \%)$ & 0.9803 \\
\hline CLIF-C ACLF score & $47.1(8.6)$ & $57.4(9.6)$ & $<0.0001$ \\
\hline MELD score & $26.5(7.2)$ & $31.3(7.2)$ & $<0.0001$ \\
\hline \multicolumn{4}{|l|}{ Laboratory data: } \\
\hline Hematocrit (\%) & $27(5)$ & $26(5)$ & 0.2100 \\
\hline Platelet count $\left(10^{9} / \mathrm{L}\right)$ & $91(71)$ & $86(68)$ & 0.5484 \\
\hline Serum bilirubin (mg/dl) & $10.6(10.9)$ & $15.2(12.5)$ & 0.0018 \\
\hline $\begin{array}{l}\text { International normalized } \\
\text { ratio }\end{array}$ & $2.1(1.0)$ & $2.4(0.9)$ & 0.0353 \\
\hline $\begin{array}{l}\text { Alanine aminotransferase } \\
(\mathrm{U} / \mathrm{L})\end{array}$ & $33(19-54)$ & $42(28-78)$ & 0.0570 \\
\hline $\begin{array}{l}\text { Aspartate aminotransferase } \\
\text { (U/L) }\end{array}$ & $64(36-107)$ & $103(55-194)$ & 0.0237 \\
\hline $\begin{array}{l}\text { Y-Glutamyltransferase } \\
(\mathrm{U} / \mathrm{L})\end{array}$ & $72(37-138)$ & $76(26-151)$ & 0.4594 \\
\hline Serum creatinine $(\mathrm{mg} / \mathrm{dl})$ & $1.9(1.2)$ & $2.4(1.5)$ & 0.0022 \\
\hline Serum sodium (mmol/L) & $135.3(6.3)$ & $134.6(6.8)$ & 0.3870 \\
\hline \multicolumn{4}{|l|}{ ACLF grade: } \\
\hline ACLF-1 & $135(54.9 \%)$ & $37(35.9 \%)$ & \\
\hline ACLF-2 & $97(39.4 \%)$ & $33(32.0 \%)$ & \\
\hline ACLF-3 & $14(5.7 \%)$ & $33(32.0 \%)$ & $<0.0001$ \\
\hline Administration of NSBBs & $124(50.4 \%)$ & $40(38.8 \%)$ & 0.0482 \\
\hline
\end{tabular}

Data are $\mathrm{n}(\%)$, mean (standard deviation) or median (Inter-quartile range). NSBBs, non-selective beta blockers.

that NSBBs provides survival advantage independently in ACLF patients.

The recent study by Gustot et al. [29] highlights the dynamic nature of ACLF suggesting that patients can move rapidly from any grade of ACLF to its resolution from whatever severity. Conversely, patients with earlier stages can deteriorate to the more advanced stages. This dynamic nature is best observed in the first week after hospital admission and the prognostic value of this observation is reflected in the CLIF-C ACLF score, where the prediction of outcome improves progressively during the first week. The clinical implication of this dynamic nature of the ACLF syndrome is the opportunity to intervene, select patients for liver transplantation and consider futility of treatment. In ACLF patients of all severities, complete resolution to no ACLF within 1-week was observed in more patients treated with NSBBs compared with those not treated, which did not reach statistical significance. However, significantly more of the patient not treated with NSBBs deteriorated to the more advanced stages.

A potential limitation of this study is that it was not specifically designed to address the role of NSBB, thus lacking data about compliance and specific reasons why NSBBs were initiated and stopped but the large prospective study design, robust data collection and analysis reduces the impact of this important limitation. The lack of data regarding refractory ascites classification limits conclusions about the use of NSBBs but the complete lack of even a signal of a deleterious outcome, the similarity in the rates of renal dysfunction and overall improved survival with NSBBs provides confidence in the conclusion that NSBBs are likely to be safe even in this population.

In conclusion, the data presented in this study suggests that ongoing administration of NSBBs are safe in cirrhotic patients developing ACLF and its use is associated with improved survival. The result of this study has not been able to confirm any deleterious effect of NSBBs in patients with ascites, SBP or alcoholic hepatitis. The mechanism it works through is the modulation of systemic inflammatory responses, which is a key pathophysiological derangement observed in ACLF patients but the exact mechanisms need to be elucidated. NSBBs provide a safe, cheap and effective therapy for patients with cirrhosis and until more prospective data are available, careful thought should be given to stopping this drug as it reduces mortality if patients go on to develop ACLF. 


\section{JOURNAL OF HEPATOLOGY}

Table 4. Relationship between white cell count, the severity of ACLF and the use of NSBBs. (A) WCC levels (mean and SD) by grade of the first ACLF episode in patients with and without BBs. (B) WCC levels (mean and SD) by worsening of the first ACLF grade after 3-7 days in patients with and without BBs. (C) WCC levels (mean and SD) by worsening of the first ACLF grade at the last in-hospital visit in patients with and without BBs.

A

\begin{tabular}{|c|c|c|c|c|c|c|c|c|c|}
\hline ACLF grade & \multicolumn{3}{|c|}{ Patients w/out NSBBs } & \multicolumn{3}{|c|}{ Patients with NSBBs } & \multicolumn{3}{|c|}{$p$ value between groups } \\
\hline ACLF-1 & \multicolumn{3}{|l|}{$9.0(6.4)$} & \multicolumn{3}{|l|}{$7.2(4.9)$} & \multicolumn{3}{|l|}{0.0824} \\
\hline ACLF-2 & \multicolumn{3}{|l|}{$11.9(6.6)$} & \multicolumn{3}{|l|}{$8.6(5.4)$} & \multicolumn{3}{|l|}{0.0042} \\
\hline ACLF-3 & \multicolumn{3}{|l|}{$12.1(6.3)$} & \multicolumn{3}{|l|}{$13.5(8.2)$} & \multicolumn{3}{|l|}{0.5428} \\
\hline & \multicolumn{3}{|l|}{ All patients } & \multicolumn{3}{|c|}{ Patients w/out bacterial infections } & \multicolumn{3}{|c|}{ Patients with bacterial infections } \\
\hline ACLF after 3-7 days & No NSBBs & NSBBs & $p$ value & No NSBBs & NSBBs & $p$ value & No NSBBs & NSBBs & $p$ value \\
\hline \multirow[t]{2}{*}{ No change/improved } & $9.9(6.4)$ & $8.8(4.3)$ & 0.1920 & $9.0(5.9)$ & $8.2(6.1)$ & 0.4196 & $12.2(7.0)$ & $10.1(6.4)$ & 0.2043 \\
\hline & \multicolumn{3}{|l|}{ All patients } & \multicolumn{3}{|c|}{ Patients w/out bacterial infections } & \multicolumn{3}{|c|}{ Patients with bacterial infections } \\
\hline $\begin{array}{l}\text { ACLF at the end of the } \\
\text { hospitalization }\end{array}$ & No NSBBs & NSBBs & $p$ value & No NSBBs & NSBBs & $p$ value & No NSBBs & NSBBs & $p$ value \\
\hline Worsened & $13.9(6.7)$ & $8.2(5.5)$ & 0.0008 & $13.0(7.3)$ & $8.1(5.8)$ & 0.0325 & $15.2(5.7)$ & $8.3(5.1)$ & 0.0086 \\
\hline
\end{tabular}

NSBBs, non-selective beta blockers.

\section{Financial support}

\section{The EASL-CLIF consortium}

The EASL-CLIF consortium is a network of 63 European university hospitals, aimed at stimulating research on pathophysiology, diagnosis and treatment of chronic liver failure. During the period 2009-2012 the consortium had received unrestricted grants from Grifols and Gambro. Grifols has prolonged its unrestricted grant for an additional period of four years. There is no other support for the consortium. The Fundació Clinic, a foundation ruled by the Hospital Clinic and University of Barcelona, administers the EASL-CLIF consortium grants. Vicente Arroyo (Chairman), Mauro Bernardi (Vice-Chairman), and members of the steering committee have no relationship with Grifols or Gambro other than conferences at international meetings (from which they may receive an honorarium) or as investigators on specific projects unrelated to the consortium. Up to now the EASL-CLIF consortium has not performed any study promoted by pharmaceutical companies. The scientific agenda of the EASL-CLIF consortium and the specific research protocols are made exclusively by the steering committee members without any participation of pharmaceutical companies.

PG received a grant from the Fondo de Investigación Sanitaria Carlos III - co financed by FEDER: FIS_PI12/00330.

\section{Conflict of interest}

Rajiv Jalan has served on Scientific Advisory Board for Conatus Pharma, has received lecture fees from Gambro and has ongoing research collaboration with Gambro, Grifols and is the principal investigator of an industry sponsored study (Sequana Medical). $\mathrm{He}$ is also inventor for a drug, L-ornithine phenyl acetate (OCR002) which UCL has licensed to Ocera Therapeutics. He is also the founder of UCL spin-out company Yaqrit ltd. and Cyberliver ltd.
Pere Ginès has received speaker honorarium and research funding from Grifols, served on the scientific advisory board for Ferring and Sequana and received research funding from Sequana.

\section{Authors' contributions}

RJ / VA / RPM: Conceived the idea, designed the protocol and wrote the manuscript.

MP: Performed all the analyses for the study.

KLT / GM / JM: Helped write the manuscript and intellectually refine the protocol.

FB, MC, JS, PG, RM: Critical review of the protocol, manuscript writing and revision.

\section{Supplementary data}

Supplementary data associated with this article can be found, in the online version, at http://dx.doi.org/10.1016/j.jhep.2015.10. 018 .

\section{References}

[1] Jalan R, Williams R. Acute-on-chronic liver failure: pathophysiological basis of therapeutic options. Blood Purif 2002;20:252-261.

[2] Moreau R, Jalan R, Gines P, et al. Acute-on-chronic liver failure is a distinct syndrome that develops in patients with acute decompensation of cirrhosis. Gastroenterology 2013;144:1426-1437, 37.e1-e9.

[3] Jalan R, Pavesi M, Saliba F, et al. The CLIF Consortium Acute Decompensation score (CLIF-C ADs) for prognosis of hospitalised cirrhotic patients without acute-on-chronic liver failure. J Hepatol 2015;62:831-840.

[4] Jalan R, Saliba F, Pavesi M, et al. Development and validation of a prognostic score to predict mortality in patients with acute-on-chronic liver failure. J Hepatol 2014:61:1038-1047.

[5] Jalan R, Stadlbauer V, Sen S, Cheshire L, Chang YM, Mookerjee RP. Role of predisposition, injury, response and organ failure in the prognosis of patients 


\section{Research Article}

with acute-on-chronic liver failure: a prospective cohort study. Crit Care 2012;1:R227.

[6] Mookerjee RP, Sen S, Davies NA, Hodges SJ, Williams R, Jalan R. Tumour necrosis factor alpha is an important mediator of portal and systemic haemodynamic derangements in alcoholic hepatitis. Gut 2003;52: $1182-1187$.

[7] Naveau S, Chollet-Martin S, Dharancy S, Mathurin P, Jouet P, Piquet MA, et al A double-blind randomized controlled trial of infliximab associated with prednisolone in acutealcoholic hepatitis. Hepatology 2004;39:1390-1397.

[8] Arroyo V, Moreau R, Jalan R. Acute-on-chronic liver failure: a new syndrome that will re-classify cirrhosis. J Hepatol 2015;62:S131-S143.

[9] Sersté T, Melot C, Francoz C, Durand F, Rautou PE, Valla D, et al. Deleterious effects of beta-blockers on survival in patients with cirrhosis and refractory ascites. Hepatology 2010;52:1017-1022.

[10] Sersté T, Francoz C, Durand F, Rautou PE, Melot C, Valla D, et al. Betablockers cause paracentesis-induced circulatory dysfunction in patients with cirrhosis and refractory ascites: a cross-over study. J Hepatol 2011:55:794-799.

[11] Mandorfer M, Bota S, Schwabl P, Bucsics T, Pfisterer N, Kruzik M, et al Nonselective $\beta$ blockers increase risk for hepatorenal syndrome and death in patients with cirrhosis and spontaneous bacterial peritonitis. Gastroenterology 2014;146:1680-1690.

[12] Sersté T, Njimi H, Degré D, Deltenre P, Schreiber J, Lepida A, et al. The use of beta-blockers is associated with the occurrence of acute kidney injury in severe alcoholic hepatitis. Liver Int 2015;35:1974-1982.

[13] Leithead JA, Rajoriya N, Tehami N, Hodson J, Gunson BK, Tripathi D, et al. Non-selective $\beta$-blockers are associated with improved survival in patients with ascites listed for liver transplantation. Gut 2015;64:1111-1119.

[14] de Franchis RBaveno V Faculty. Revising consensus in portal hypertension: report of the Baveno $\mathrm{V}$ consensus workshop on methodology of diagnosis and therapy in portal hypertension. J Hepatol 2010;53:762-768.

[15] Lebrec D, Nouel O, Bernuau J, Bouygues M, Rueff B, Benhamou JP. Propranolol in prevention of recurrent gastrointestinal bleeding in cirrhotic patients. Lancet 1981;1:920-921.

[16] Ge PS, Runyon BA. Beta-blockers in cirrhosis: thank you for your attention. J Hepatol 2014;61:451-452.

[17] Madsen BS, Havelund T, Krag A. Targeting the gut-liver axis in cirrhosis: antibiotics and non-selective $\beta$-blockers. Adv Ther 2013;30:659-670.
[18] Morelli A, Ertmer C, Westphal M, Rehberg S, Kampmeier T, Ligges S, et al. Effect of heart rate control with esmolol on hemodynamic and clinical outcomes in patients with septic shock: a randomized clinical trial. JAMA 2013;310:1683-1691.

[19] Worlicek M, Knebel K, Linde HJ, Moleda L, Schölmerich J, Straub RH, et al. Splanchnic sympathectomy prevents translocation and spreading of E coli but not S aureus in liver cirrhosis. Gut 2010;59:1127-1134.

[20] Schäper J, Wagner A, Enigk F, Brell B, Mousa SA, Habazettl H, et al. Regional sympathetic blockade attenuates activation of intestinal macrophages and reduces gut barrier failure. Anesthesiology 2013;118:134-142.

[21] Elenkov IJ, Iezzoni DG, Daly A, Harris AG, Chrousos GP. Cytokine dysregulation, inflammation and well-being. Neuroimmunomodulation 2005;12:255-269.

[22] Pecivová J, Macicková T, Lojek A, Gallová L, Cíz M, Nosál' R, et al. Effect of carvedilol on reactive oxygen species and enzymes linking innate and adaptive immunity. Neuro Endocrinol Lett 2006:160-163.

23] Shah N, Dhar D, El Zahraa Mohammed F, Habtesion A, Davies NA, JoverCobos $\mathrm{M}$, et al. Prevention of acute kidney injury in a rodent model of cirrhosis following selective gut decontamination is associated with reduced renal TLR4 expression. J Hepatol 2012:56:1047-1053.

[24] Fernández J, Navasa M, Planas R, Montoliu S, Monfort D, Soriano G, et al. Primary prophylaxis of spontaneous bacterial peritonitis delays hepatorenal syndrome and improves survival in cirrhosis. Gastroenterology 2007:133:818-824.

[25] Bass NM, Mullen KD, Sanyal A, Poordad F, Neff G, Leevy CB, et al. Rifaximin treatment in hepatic encephalopathy. N Engl J Med 2010:362:1071-1081.

[26] Senzolo M, Cholongitas E, Burra P, Leandro G, Thalheimer U, Patch D, et al. Beta-Blockers protect against spontaneous bacterial peritonitis in cirrhotic patients: a meta-analysis. Liver Int 2009;29:1189-1193.

[27] Kimer N, Feineis M, Møller S, Bendtsen F. Beta-blockers in cirrhosis and refractory ascites: a retrospective cohort study and review of the literature. Scand J Gastroenterol 2015;50:129-137.

[28] Bajaj JS, Ratliff SM, Heuman DM, Lapane KL. Non-selective beta-blockers are not associated with serious infections in veterans with cirrhosis. Aliment Pharmacol Ther 2013:38:407-414.

[29] Gustot T, Fernandez J, Garcia E, et al. Clinical course of acute-on-chronic liver failure and effects on prognosis. Hepatology 2015;62:243-252. 